\title{
THE FIXED POINT INDEX FOR ACCRETIVE MAPPING* WITH K-SET CONTRACTION PERTURBATION IN CONES
}

\author{
YU-QING CHEN \\ Department of Mathematics \\ Sichuan University \\ 610064 Chengdu \\ P. R. China \\ (Received July 21, 1993 and in revised form August 15, 1994)
}

ABSTRACT : Let $P$ be a cone in Banach space E. A, K are two mappings in $P, A$ is accretive, $K$ is $k-$ set contraction. then a fixed point index is defined for mapping $-A+K$. some fixed point theorems are also deduced.

KEY WORDS AND PHRAESE : accretive mapping, $\mathbf{k}$ - set contraction, cone, fixed point index.

1992 AMS SUBJECT CLASSIFICATION CODES : 47H10. 47H05. 54H25

\section{INTRODUCTION}

The fixed point index is a important tool in solving positive solutions of nonlinear equations in ordered Banach space. So what nonlinear mapping could be defined a index theory becomes a very interesting problem, many authors have studied this problem. see[1].[2].[8].[10], [12].[13]. In this paper. $E$ is a Banach space , $r \subset E$ is a closed cone, i. e $\mathrm{P}$ is closed convex, and

$$
\lambda P \subset P, \forall \lambda \geqslant 0, P \cap(-P)=\{0\} ;
$$

$\Omega \subset E$ is a nonempty open bounded subset. Let $A: D(A) \subset P \rightarrow 2^{\rho}$ be a multivalued accretive mapping. $\mathrm{i}$, $\mathrm{e}$

$K: \bar{\Omega} \cap P \rightarrow P$ is a strict $\mathbf{k}-$ set contraction. i,e $0 \leqslant k<1$; If

$$
\|x-y\| \leqslant\left\|x-y+\lambda\left(a_{1}-a_{2}\right)\right\|, x, y \in D(A), a_{1} \in A x, a_{2} \in A y ;
$$

$$
(I+A)(D(A))=P \text {, and } x \notin-A x+K x, \forall x \in a \Omega \cap D(A),
$$

then a fixed point index is defined for $-A+K$, when $\mathrm{K}$ is compact, such type mapping were studied by $[4],[5]$, $[14],[15]$.

\section{MAIN RESULTS}

Let $\mathrm{E}$ be a Banach space, $P \subset E$ is a closed cone, $" \leqslant "$ is the order induced by $\mathrm{P}$ in $\mathrm{E}, \mathrm{i}, \mathrm{e} x \leqslant y$ if and only if $y$ $-x \in P$

PROPOSITION 1: $A: D(A)=P \rightarrow P$ is a continuous accretive mapping, for each $x \in P$, there exists $\beta(x)>0$, such that $A x \leqslant \beta(x), x$, then $(\lambda I+A) P=P, \forall \lambda>0$;

PROOF. : For each $z \in P$, consider the following differential equation

$$
\left\{\begin{array}{l}
x^{\prime}(t)=-(\lambda I+A) x(t)+z, t \in[0,+\infty) \\
x(0)=u \in P
\end{array}\right.
$$

For each $\mathrm{x} \in \mathrm{P}$. sunce $A x \leqslant \beta(x) \cdot x$, so there exists $\mathrm{W}(\mathrm{x}) \in \mathrm{P}$, such that $\beta(x) \cdot x=A x+W(x)$

So we have $x+\varepsilon(-\lambda x-A x+z)=(1-\varepsilon \lambda-\varepsilon \beta(x)) x+\varepsilon W(x)+\varepsilon z$ For sufficient small $\varepsilon>0$, such that $1-\varepsilon \lambda-\varepsilon \beta(x)>0$, then $(1-\varepsilon \lambda-\varepsilon \beta(x)) x+\varepsilon W(x)+\varepsilon z \in P$ Hence

$$
\lim _{\operatorname{lin}_{-}} \frac{1}{\varepsilon} \rho(x+\varepsilon(-\lambda x-A x+z), P)=0, \forall x \in P
$$


by [6]. we know (E1) has only one solution. Let $x(t, u)$ be the unique solution of $\left(E_{1}\right)$ with $x(0)=u$.

Now. defune a mapping $B_{1}: P \rightarrow P$ as following

$$
B_{1} u=x(T, u), u \in P, T>0 \text { is a constant; }
$$

For $u, v \in P$. Let $\varnothing(t)=\|x(t, u)-x(t, v)\|$, then

$\varnothing(t) D \varnothing(t) \leqslant\left(x^{\prime}(t, u)-x^{*}(t \cdot v) \cdot x(t \cdot u)-x(t \cdot v)\right)$.

where $D \varnothing(t)=\overline{l i m}_{h \rightarrow 0^{-}} \frac{\varnothing(t)-\varnothing(t-h)}{h}$; see ([6]P.36)

$$
\varnothing(t) D \varnothing(t) \leqslant(-\lambda x(t, u)-A x(t, u)+\lambda x(t, v)+A x(t, v), x(t, u)-x(t, v))
$$

$A$ is accertive. so

Therefore

$$
(-A x(t, u)+A x(t, v) \cdot x(t, v)-x(t, v))=-(A x(t, u)-A x(t, v), x(t, u)-x(t, v))_{-} \leqslant 0
$$

$$
\begin{gathered}
\varnothing(t) D \varnothing(t) \leqslant-\lambda \varnothing^{2}(t) \\
\varnothing(t) \leqslant p{ }^{x t} \varnothing(0)
\end{gathered}
$$

So we have $\left\|B_{1} u-B_{1} v\right\| \leqslant e^{x t}\|u-v\|$

Hence, $\mathrm{B}_{\mathrm{T}}$ has a unique fixed point $u_{0} \in P, t, e B_{t} u_{0}=u_{\mathrm{n}}$. This implies $x^{\prime}\left(t, u_{0}\right)=0, t>0$,

So $0=-\lambda u_{0}-A u_{0}+z, z \in(A+\lambda I)(P)$.

This complete the proof.

In the following, we assume $A: D(A) \subset P \rightarrow 2^{P}$ is a multivalued accritive mapping, $(A+I)(D(A))=P$, It's well known $(I+A){ }^{\prime}$ is nonexpansive ( $\left.\operatorname{see}[4]\right)$.

Let $\Omega$ be a open bounded subset of $E, K: \bar{\Omega} \cap P \rightarrow P$ is a strict $\mathbf{k}$ - set contraction, i, e $k \in[0,1)$;

Suppose $D(A) \cap \bar{\Omega} \neq \varnothing$, and $x \notin-A x+K x, \forall x \in \Omega \Omega \cap D(A)$, then

$$
x \neq(I+A){ }^{\prime} K x, \forall x \in a \Omega \cap P \text {; }
$$

$(I+A){ }^{1} K$ is also a strict $\mathbf{k}$ - set contraction. so the fixed point index $i\left((I+A)^{-1} K, \Omega \cap P\right)$ is well defined, see $[1],[8]$. Now, we define

$$
\iota(-A+K, \Omega \cap D(A))=i\left((I+A){ }^{1} K, \Omega \cap P\right)
$$

THEOREM 1: (a) If $\Omega=B(0, r), K x=x_{0} \in B(0, r) \cap P, \forall x \in B(0, r) \cap P$, then

$$
\text { , }(-A+K, B(0, r) \cap D(A))=1
$$

(b) Suppose $\Omega=\Omega_{1} \cup \Omega_{2}, \Omega_{1} \cap \Omega_{2}=\varnothing$, then

$$
i(-A+K, \Omega \cap D(A))=i\left(-A+K \cdot \Omega_{1} \cap D(A)\right)+\imath\left(-A+K, \Omega_{2} \cap D(A)\right)
$$

(c) Let $H(t, x):[0,1] \times(\bar{\Omega} \cap P) \rightarrow P$. if $\mathrm{H}(\mathrm{t}, \mathrm{x})$ is uniformly continuous in $\mathrm{x}$ for each $\mathrm{t}$, and for each $t \in[0,1] . H$ $(t, \cdot): \bar{\Omega} \cap P \rightarrow P$ is a strict $\mathrm{k}$ - set contraction, $\mathrm{k}$ doesn't depend on $\mathrm{t}$, suppose $x \notin-A x+H(t, x), \forall x \in a \Omega \cap D(A), t \in[0.1] ;$

then $i(-A+H(t, x), \Omega \cap D(A))$ doesn't depend on t.

(d) If $i(-A+K, \Omega \cap D(A)) \neq 0$, then $x \in-A x+K x$ has a solution in $\Omega \cap D(A)$. i,e $-A+K$ has a fixed point. PROOF : by the definition, (b), (c), (d) is obvious. (see[1]or [8])

Now, we prove (a). First, we have

$$
0 \in D(A) \text {, and } 0 \in A 0
$$

In fact, $(A+I) D(A)=P$, so there exists $x \in D(A), a \in A x$, such that $x+a=0$.

Since $x \geqslant 0 . a \geqslant 0$, So we must have $x=0, a=0 \in A 0$. Hence

$$
(A+I)^{\prime} 0=0
$$

by the definition, we need to prove

$$
i\left((I+A)^{-1} K, \Omega \cap P\right)=1, \Omega=B(0, r)
$$

Since $(I+A){ }^{1} K x=(I+A){ }^{1} x_{0}, \forall x \in \bar{\Omega} \cap P$, and

$$
\left\|(I+A)^{-1} x_{0}-(I+A)^{-1} 0\right\| \leqslant\left\|x_{0}\right\|<r
$$

So $(I+A){ }^{1} x_{0} \in \Omega \cap P=B(0, r) \cap P$, by $[1]$ (see also[8]).

$$
{ }_{t}\left((I+A)^{-1} K, B(0 . r) \cap P\right)=1
$$

So $i=(-A+K, B(0, r) \cap D(A))=1$.

In the following, $K, A, \Omega$, are same as above.

LEMMA 1 : If $K x \geq x, \forall x \in \Omega \Omega \cap P$; and $0 \in \Omega$, then

$$
i(-A+K \cdot \Omega \cap D(A))=1
$$

PROOF : Let $H(t, x)=t K x, t \in[0,1], x \in \bar{\Omega} \cap P$. If $x \in-A x+t K x$ for some $x \in \Omega \Omega \cap D(A)$ and $t \in[0,1]$, 
then $t \neq 0$ (otherwise, we gct $\mathrm{z}=0 \in \Omega \Omega$. a contradiction )

So $K . x \geqslant \frac{x}{t} \geqslant x$. a contradiction to $K x \geqslant x$.

Hence. $H(t \cdot x)$ satisfy all the conditions of (c) in theorem 1 .

So

$$
\text { ( }(-A+K \cdot \Omega \cap D(A))=\imath(-A+0 . \Omega \cap D(A))
$$

by (2.3). we have $(I+A) \quad$ ' $0=0 \in \Omega \cap I^{\prime}$

So $\left.(I+A){ }^{\prime} 0 . \Omega \cap P\right)=1$, and we get

Hence

$$
(1-A+0 . \Omega \cap D(A))=1
$$

$$
i(-A+K \cdot \Omega \cap D(A))=1
$$

COROLLARY 1: If $0 \in \Omega$, and $K x<x \cdot \forall x \in a \Omega \cap P$, then $-A+K$ has a fixed point in $\Omega \cap D(A)$

PROOF : It's obvious $K x x \cdot \forall x \in a \Omega \cap P$. By lemma 1 .

$$
\text { ( }(-A+K \cdot \Omega \cap D(A))=1
$$

Theorem 1. (d) implies $-A+K$ has a fixed point in $\Omega \cap D(A)$.

LEMMA 2: Let $\mathrm{u}_{1} \neq 0, \mathrm{u}_{n} \in \mathrm{P}$. suppose $x-t u_{0} \notin-A\left(x-t u_{0}\right)+K x$, if $x \in 2 \Omega \cap P$, and $x-t u_{0} \in D(A)$, for $\mathrm{t} \geqslant$ 0; Then

$$
\text { ( }(-A+K, \Omega \cap D(A))=0
$$

PROOF: Suppose $\imath(I+A){ }^{1} K \cdot \Omega \cap D(A) \neq 0$

For each $\tau>0$. Let $H(t, x)=(I+A)^{\prime} K+t \tau u_{1}, \forall x \in \Omega \cap P, t \in[0,1]$;

It's obvious $\mathrm{H}(\mathrm{t}, \mathrm{x})$ is uniformly continuous in $\mathrm{x}$ for each $\mathrm{t}$, and $\mathrm{H}(\mathrm{t}, \cdot)$ is strict $\mathrm{k}$ - set contraction for each $\mathrm{t}$. $B y[1]$. (see also [8]). We get

$$
\left.\iota\left((I+A){ }^{\prime} K+\tau u_{0}, \Omega \cap P\right)={ }_{\imath}(I+A){ }^{\prime} K . \Omega \cap P\right) \neq 0
$$

So there exists $x_{r} \in \Omega \cap P$, such that

$$
x_{\mathrm{r}}-(I+A){ }^{1} K x_{\mathrm{r}}=\tau u_{0}
$$

Letting $\tau \rightarrow \infty$, the left side of (2.6) is bounded, but the right side of $(2,6)$ is unbounded, a contradiction.

We must have $t(-A+K, \Omega \cap D(A))=0$

THEOREM 2:If $A: D(A) \subset P \rightarrow 2^{p}$ is an accretive mapping, $(I+A) D(A)=P, \Omega_{1}, \Omega_{2}$ are two open bounded subsets of $E .0 \in \Omega_{1} \subset \Omega_{2}, K: \bar{\Omega} \cap P \rightarrow$ is a strict $\mathrm{k}$ - set contraction mapping, $0 \neq u_{0} \in P$

(i) For each $x \in 2 \Omega_{2}, x \not K x$; for each

$$
x \in a \Omega_{1} \cap P, x-t u_{0} \in D(A), t \geqslant 0 . x-t u_{0} \notin-A\left(x-t u_{0}\right)+K x ;
$$

(ii) For each $x \in 2 \Omega_{1} ; x \not K x$, for each

$$
x \in a \Omega_{2} \cap P, x-t u_{0} \in D(A), t \geqslant 0, x-t u_{0} \notin-A\left(x-t u_{0}\right)+K x ;
$$

Suppose either (i)or (ii) is satisfied, then $-\mathrm{A}+\mathrm{K}$ has a fixed point in $\left(\Omega_{2}-\bar{\Omega}_{1}\right) \cap D(A)$

PROOF : Suppose condition (i) is satısfied by, Lemma 1 , we have

$$
i\left(-A+K, \Omega_{2} \cap D(A)\right)=1
$$

by Lemma 2 , we have

$$
i\left(-A+K, \Omega_{1} \cap D(A)\right)=1
$$

by (b) of Theorem 1 , and (6), (7). We get

$$
i\left(-A+K,\left(\Omega_{2}-\bar{\Omega}_{1}\right) \cap D(A)\right)=1
$$

by (d) of Theorem 1, we know $-A+K$ has a fixed point in $\left(\Omega_{2}-\bar{\Omega}_{1}\right) \cap D(A)$.

If (ii) is satisfied, the proof is sımilar. We complete the proof.

THEOREM 3: For each $x \in \Omega \Omega \cap D(A),\|K x\| \leqslant\|x\|$, and $0 \in \Omega$, then $-A+K$ has a fixed point in $\bar{\Omega} \cap D$ (A)

PROOF : we may suppose

$$
x \notin-A x+K x \cdot \nabla \quad \checkmark \in D(A)
$$

Let $H(t, x)=t K x, \forall x \in a \Omega \cap P, t \in[0,1]$;

It's obvious $\mathrm{H}(\mathrm{t}, \mathrm{x})$ is uniformly continuous in $\mathrm{x}$, and $\mathrm{H}(\mathrm{t}, \cdot)$ is strict $\mathbf{k}$-set contraction for each $\mathrm{t}$.

We show that

$$
x \notin-A x+H(t, x), \forall x \in a \Omega \cap D(A), t \in[0,1]
$$

If $x \in-A x+H(t, x)$ for some $x \in \Omega \Omega \cap D(A), t \in[0,1]$, then $x=(I+A){ }^{\prime} H(t, x)$ 
Since $(I+A)$ ' is nonexpansive and $(I+A)^{1} 0=0$. So

$$
\|x\| \leqslant\|H(t \cdot x)\|=\|t K x\| \leqslant t\|x\|
$$

Therefore $\mathrm{t}=1$, contradict to $(8)$, by $(\mathrm{c})$ of Theoreml.

$$
\iota(-A+K . \Omega \cap D(A))=\imath(-A+0, \Omega \cap D(A))
$$

and (2.5) implies $t(-A+K, \Omega \cap D(A))=1$.

by (d) of Theorem $1,-A+K$ has a fixed point in $\Omega \cap D(A)$.

THEOREM 4:If $0 \in \Omega .\|K x\| \leqslant\|x+a\|, \forall x \in a \Omega \cap D(A), a \in A x$; then $-A+K$ has a fixed point in $\bar{\Omega} \cap$ $D(A)$.

PROOF: We may assume $x \in-\Lambda x+K x, \forall x \in 2 \Omega \cap D(A)$;

Let $H(t, x)=t K x, t \in[0,1], x \in \bar{\Omega} \cap P$;

If $x \in-A x+t K x$ for some $t \in[0.1], x \in a \Omega \cap D(A)$, then $t K x \in x+A x$

So there exists $a \in A x$, such that $t K x=x+a$. We have $\|K x\| \leqslant t\|K x\|$

By the assumption (2.11), $t \neq 1$, we must have $K x=0, x+a=0$

By (2.3), $x=0 \in \Omega \Omega$, a contradiction to $0 \in \Omega$.

So we have $x \in-A x+H(t, x), \forall x \in a \Omega \cap D(A), t \in[0,1]$.

The following proof is similar to that of Theorem 3. This end the proof.

\section{REFERENCES}

1. H. AMMAN, On the number of solutions of nonlinear equations in ordered Banach spaces, J. Funct. Anal. 11 (172). $346-384$

2: H. AMMAN, Fixed point equations and nonlinear eigenvalue problems in ordered Banach spaces. SIAM, Rev, 18 (1976) 620-709

3. F, E. BROWEDR, Nonlinear mappings of nonexpansive and accretive type in Banach spaces. Bull. Amer. Math. Soc. 73(1967)875-882

4. F,E. BROWDER, Nonlinear operators and nonlinear equations of evolution in Banach spaces. Proc. Symp. Pure. Math. V18,part2. AMS 1976

5. Y, Z. CHEN. The generalized degree for compact perturbations of $\mathrm{m}$-accretive operators and applications. Non. Anal. 13(1989)393-403

6. K. DEIMLING. Ordinary differential equations in Banach spaces. Lect. Notes. Math. 596. 1977

7. M. A. KRASNOSELSKII. Positive solutions of operator equations. Noordhoff. 1964

8. G. 7.. LI. The fixed point index and the fixed point theortnis of 1 -set-contraction mappings. Proc. Amer. Math. Soc 104 (1988). 393-1170

9. T. C. LIN. Approximation theorems and fixed point theorems in cones. Proc. Amer. Math. Soc. 102 (1988). 502506

10. P. M. FitzPatriCK, W. V. PETRYShyN. Fixed Point theorems and the fixed point index for multivalued mappings in cones. J. L. Math. Soc. 12 (1975)75-85

11. I. MASSABO,C, A. STUART. Positive eigenvectors of $k-$ set contractions. Non. Anal. $3(179) .35-44$

12. R. D. NAUSSBAUM. The fixed point index and asymptotic fixed point theorems for $\bar{k}-$ set contractions. Bull. Amer. Math. Soc. 75 (1969). 490-495.

13. R. D. NAUSSBAUM. The fixed point index for local condensing maps. Ann. Mat. Pura. Appl. 89(1992). 1-9

14. C. MORALES, on the range of sums of accretive and continuors operatous in Banach spaces. Non. Anal. 19 (1992). 1-9

15. A. G. KARTSATOS, Mapping theorems involving ranges of sums on nonlınear operators. Non. Anal. $\underline{6}$ (1982). $271-278$ 


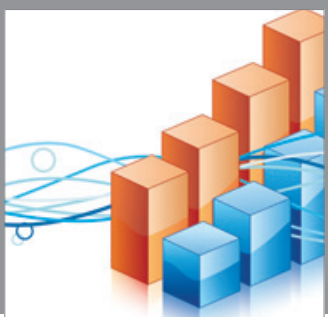

Advances in

Operations Research

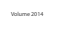

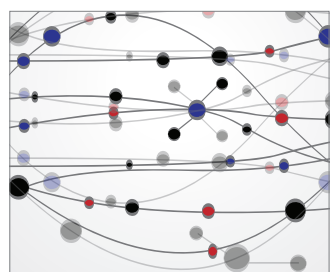

\section{The Scientific} World Journal
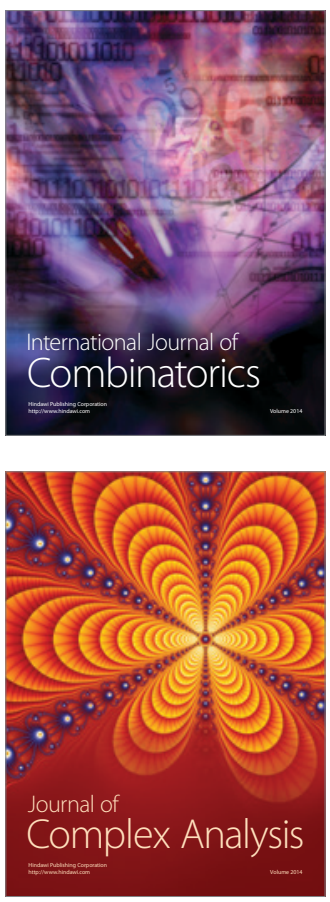

International Journal of

Mathematics and

Mathematical

Sciences
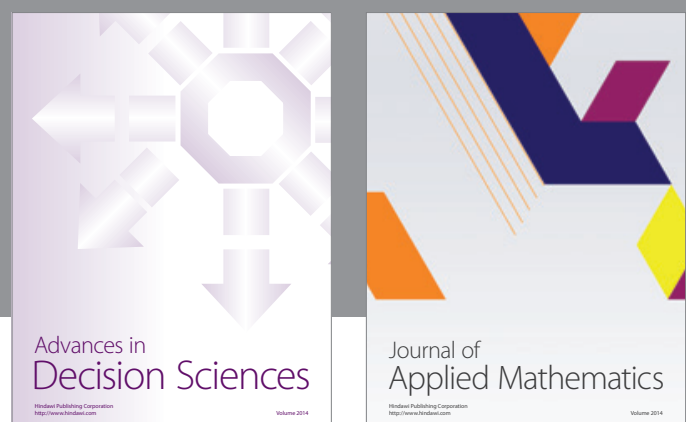

Journal of

Applied Mathematics
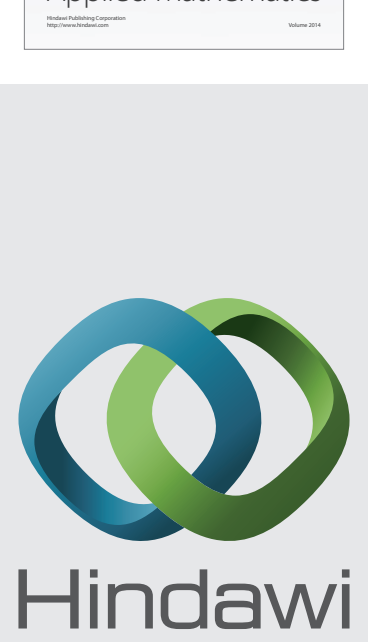

Submit your manuscripts at http://www.hindawi.com
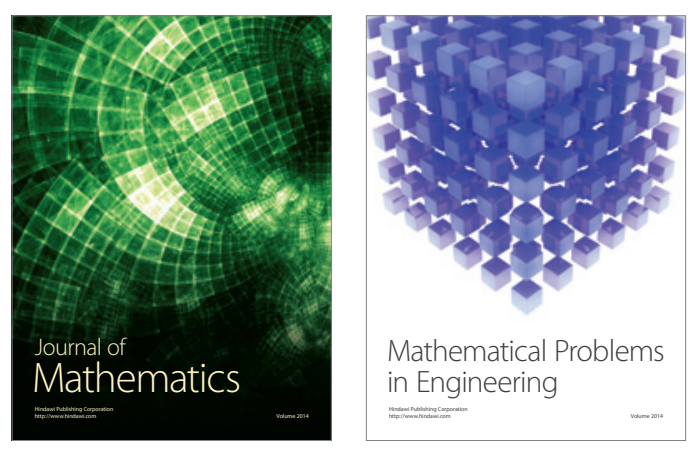

Mathematical Problems in Engineering
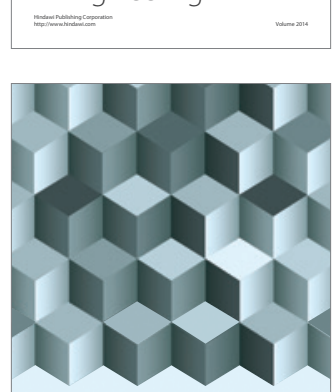

Journal of

Function Spaces
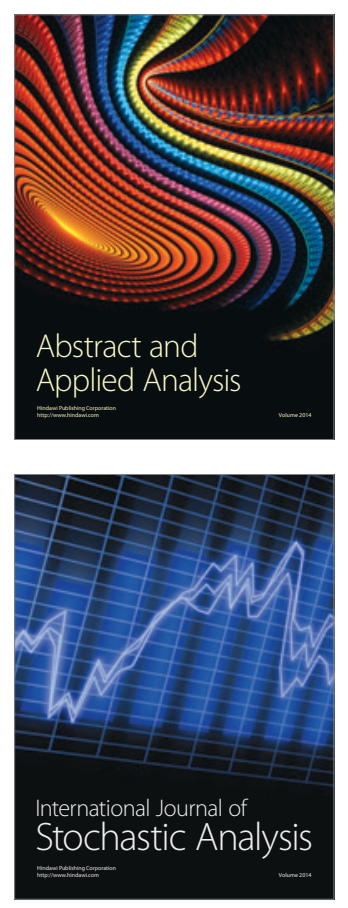

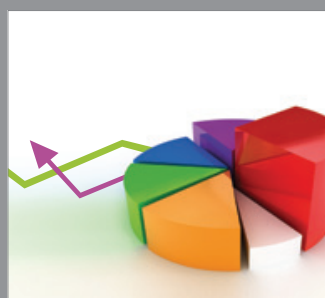

ournal of

Probability and Statistics

Promensencen
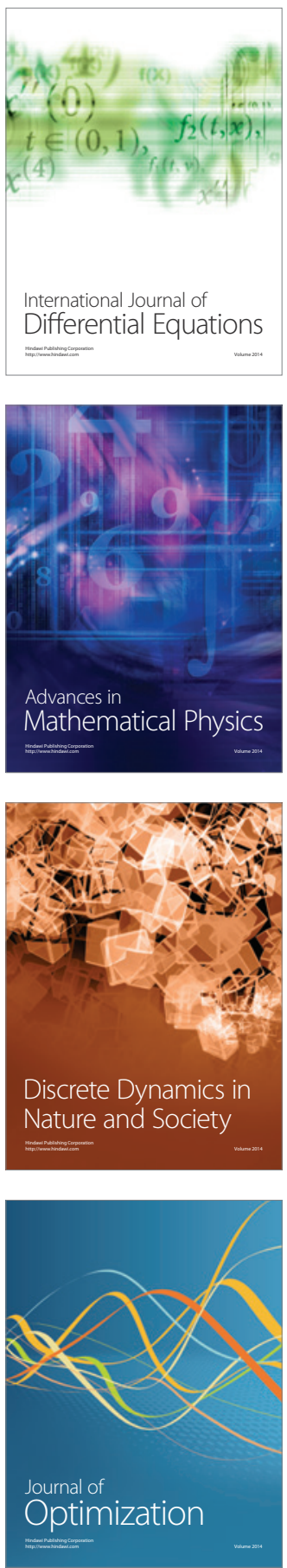